\title{
A new method for the diagnosis of power backbone communication network based on extension rating
}

\author{
$\mathrm{Li} \mathrm{Li}^{1, \mathrm{a}}$, Tiantian Luo ${ }^{2, b^{*}}$, Huiqi Shen ${ }^{1, \mathrm{c}}$, Runze $\mathrm{Wu}^{2, \mathrm{~d}}$, \\ ${ }^{1}$ State Grid Jibei Electric Power Company Economic Research \\ Institute.Fengtai,Beijing,100160,China \\ ${ }^{2}$ School of Electrical and Electronic Engineering, North China Electric Power \\ University,Changping,Beijing,102206,China \\ ajbjyyll@126.com, ${ }^{\mathrm{b}}$ shenhuiqi1989@163.com, ${ }^{\mathrm{c}} 710195397 @ q q . c o m,{ }^{\mathrm{d}}$ wrz_y@126.com
}

Keywords: Electric power communication network; an index system; extension rating; diagnose and rate;

\begin{abstract}
Electric power communication network is an important part of power system, which plays a vital role in the operation of power network. This paper established an index system of electric power communication network and proposed a diagnostic method of electric power communication network system based on extension rating. This evaluation method can not only evaluate the impact factors of the electric power communication network, but also can diagnose and rate the whole electric power communication network.
\end{abstract}

\section{Introduction}

The power system communication network is one of the important parts of the power system. It is the basis of the power system dispatching automation. The safe operation of power system communication network is important to the safety operation of power network. Electric power communication network gradually formed by cross regional, city regions, provinces (including counties), a total of four level communication network. At present, there are few studies on the overall diagnosis of power backbone communication network.

Paper [1] analyzes the important factors that influence the development of power grid from the two levels of technology and operation, and construct a regional power network development level diagnosis system. Paper [2] presents a kind of fuzzy consistent evaluation method according to the requirement of DER network monitoring communication. Analytic hierarchy process method [3] can select the best program from many alternatives but it can't evaluate a single program. Paper [4] builds the evaluation model of the functional effect of the substation construction project based on the multilevel extension evaluation method, and evaluates the effect of the project function. The method in paper [4] can evaluated the object according to the composite operation of all the indexes and their weights, but it can't reflect the overall quality of all the indexes from the whole point of view. Paper [5] builds a risk evaluation index system of photovoltaic power generation project, and uses the TOPSIS model based on grey relational degree to evaluate the risk of the program.

In this paper, a new method for the diagnosis of electric power communication network based on extension rating is presented. This method uses the interval weight to reduce the subjectivity of the index weight and applies the extension theory to the evaluation of the power communication network. It can not only make a comprehensive diagnosis and evaluation of the electric power communication network from the whole angle, but also can evaluate the key factors that effect the electric power communication network. The feasibility and rationality of the method are verified by the empirical analysis.

\section{Diagnostic Index Model of Electric Power Communication Network}

The Diagnostic Requirements of Power Communication Network. With the development of 
power network, power communication network is growing rapidly. According to state grid development planning, prefectures and counties network will integrate the four-level power communication network, municipal communication network will converge to the access network business. The construction method of relying on the experience can't meet the current development trend. Moreover, with the upgrading of the communication equipment, the mixed use of obsolete equipment and new equipment would cause failures of equipment. So the security, reliability and adaptability of the new power communication network should be reconsidered. And the analysis of the power communication network is the key to solve these problems.

Diagnostic Index Model. Through the research on the characteristics of the power communication network, this paper establishes the index system of the power communication network, including three first-level indexes: safety reliability, equipment status and service support. The safety reliability indexes include seven second-level indexes: number of business interruption $A_{1}$, number of optical transmission equipment $A_{2}$, failure rate of communication power $A_{3}$, failure time of optical transmission equipment $\mathrm{A}_{4}$, failure time of communication power $\mathrm{A}_{5}$, failure rate of cable A6,number of cable failure $A_{7}$. The equipment status indexes include five second-level indexes: the ratio of 48 core-optic cable $\mathrm{B}_{1}$, the ratio of optical transmission equipment that is used more than 10 years $B_{2}$, the length ratio of optical cable that is used more than 10 years $B_{3}$, total length of OPGW optical link $\mathrm{B}_{4}$, total length of ADSS optical link $\mathrm{B}_{5}$.The service support indexes include five second-level indexes: number of power production communication service $\mathrm{C}_{1}$, number of power information communication service $C_{2}$, the net flow of main export business $C_{3}$, the net flow of spare export business $\mathrm{C}_{4}$, municipal $10 \mathrm{kV}$ site communication coverage $\mathrm{C}_{5}$.

\section{Evaluation Model of Multilevel Extension Diagnosis Method}

Overview of Multilevel Extension Method. The extension method can obtain the membership degree of the upper level index by the complex operation of the index weight and the actual index value. And the method gave the evaluation result according to the maximum membership principle. Multilevel extension method can obtain the evaluation results by the complex combination of the upper level indexes' weights and upper level indexes' evaluation results. Its logical block diagram is shown in Fig.1.

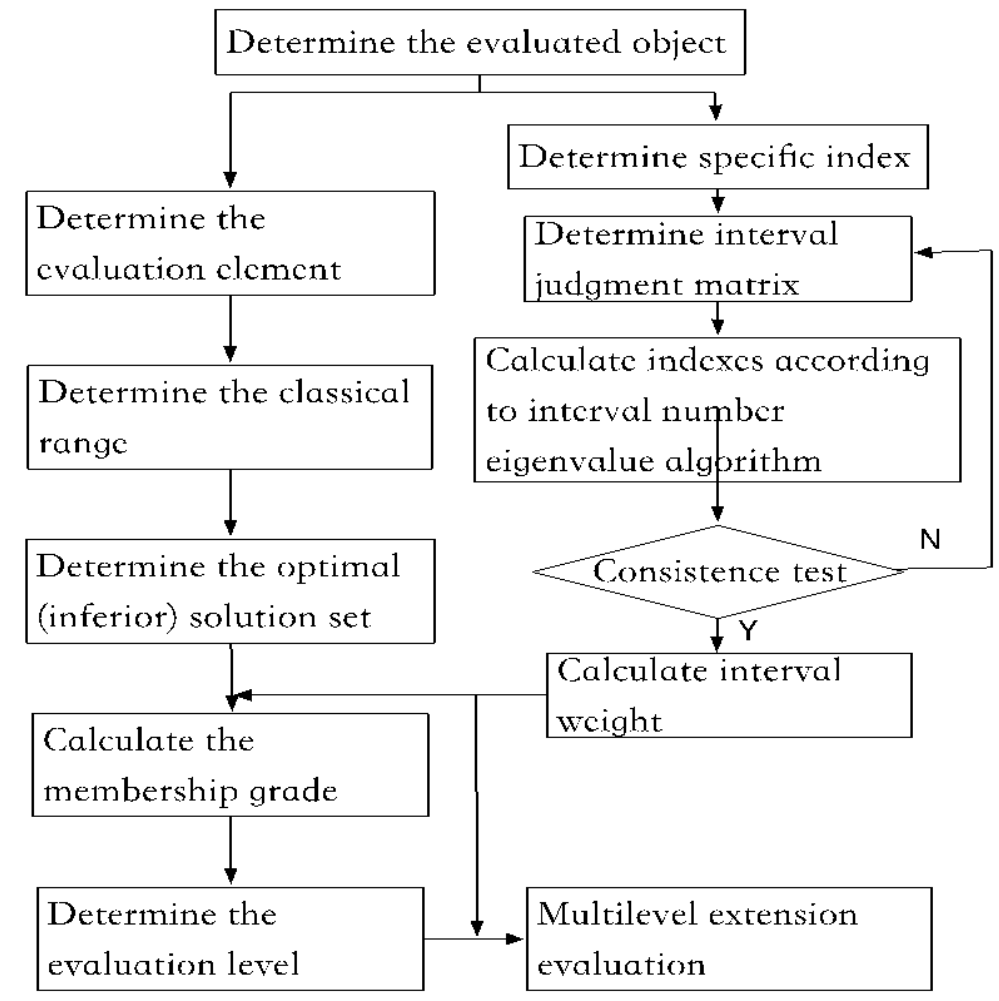

Figure 1 logical block diagram of extension 
Determine the Index Weight. According to the 1 9 scale method in Tab.1, we mark the relative importance of each two indexes. In order to eliminate the uncertainty of subjective judgment, the comparison results are expressed in the interval number. The final result of the interval number judgment matrix is shown in Eq.1.

Tab.2 $\quad 1 \sim 9$ scale rules

\begin{tabular}{|c|c|c|c|}
\hline Level & Degree & Meaning \\
\hline 1 & The same & Index a and B are equally important \\
\hline 3 & A bit & Index a is slightly more important than the index b \\
\hline 5 & Obviously & Index a is obviously more important than the index b \\
\hline 7 & Strongly; & Index a is strongly more important than the index b \\
\hline 9 & Extremely & Index a is extremely more important than the index b \\
\hline & $A=\left[\begin{array}{cccc}{[1,1]} & {\left[a_{12}^{-}, a_{12}^{+}\right]} & \ldots & {\left[a_{1 n}^{-}, a_{1 n}^{+}\right]} \\
{\left[a_{21}^{-}, a_{21}^{+}\right]} & {[1,1]} & \ldots & {\left[a_{2 n}^{-}, a_{2 n}^{+}\right]} \\
\ldots & \ldots & \ldots & \ldots \\
{\left[a_{n 1}^{-}, a_{n 1}^{+}\right]} & {\left[a_{n 2}^{-}, a_{n 2}^{+}\right]} & \ldots & {[1,1]}\end{array}\right]$
\end{tabular}

In Eq.(1), $a_{i j}^{+}$and $a_{i j}^{-}$represent the upper and lower bounds for the relative importance of index $\mathrm{i}$ and the index j. According to the arithmetic method based on interval number, there is $A=\left[A^{-}, A^{+}\right]$. $A^{+}$and $A^{-}$are the upper and lower bounds matrix.

According to the interval number eigenvalue method, the largest eigenvalue of $A^{+}$and $A^{-}$can be calculated, that are $\lambda_{\max }^{+}$and $\lambda_{\max }^{-}$. Tormalized feature vector with positive components $w^{+}$and $w^{-}$can also be calulated. Then the positive component coefficient $\mathrm{m}$ and the negative component coefficient $\mathrm{k}$ of the matrix A are calculated by using the Eq.(2) and Eq.(3).

$$
\begin{aligned}
& m=\sqrt{\sum_{j=1}^{n}\left(1 / \sum_{i=1}^{n} a_{i j}^{-}\right)} \\
& k=\sqrt{\sum_{j=1}^{n}\left(1 / \sum_{i=1}^{n} a_{i j}^{+}\right)}
\end{aligned}
$$

According to the [6], the consistency test index $C R^{+}$and $C R^{-}$can be calculated. When the value of $\frac{1}{2}\left(C R^{+}-C R^{-}\right)$is less than 0.1 , the matrix A pass conformance test. Assuming the second-level index interval weight vector is $W=\left[\mathrm{kw}^{-}, \mathrm{mw}^{+}\right]$, the first-level index interval weight vector is $\mu=\left[\mu_{1}, \mu_{2, \ldots} \mu_{k}\right]$. ( $\mu_{k}$ is interval number)

\section{Extension Rating Model.}

(1) Determine the evaluation matter

Assume the evaluation object contains $m$ indexes, that are $C_{1}, C_{2}, \cdots C_{m}$. The element model is shown in Eq.(4).

$$
M=\left(U, C_{k}, v_{k}\right)=\left[\begin{array}{ccc}
U & C_{1} & v_{1} \\
& C_{2} & v_{2} \\
& \ldots & \ldots \\
& C_{m} & v_{m}
\end{array}\right]
$$

In Eq.(4), M represents the element model. U represents the evaluation classes for the evaluation of the object. $v_{k}(\mathrm{k}=1,2, \ldots \mathrm{m})$ represent the practical value of each index.

(2) Determine the classical range 
Divide the evaluation results into $n$ levels. The classical range model is shown in Eq.(5).

$$
M_{j}=\left(U_{j}, C_{k}, v_{j k}\right)=\left[\begin{array}{ccc}
U_{j} & C_{1} & <a_{j 1}, b_{j 1}> \\
& C_{2} & <a_{j 2}, b_{j 2}> \\
\ldots & \ldots \\
& C_{m} & <a_{j m}, b_{j m}>
\end{array}\right]
$$

In Eq.(4), $M_{j}$ represents the element model of level $\mathrm{j}$. $U_{j}$ represents the evaluation result of level j. $<a_{j k}, b_{j k}>$ represent the value range of $C_{k}$ in level $\mathrm{j}$.

(3) Determine the optimal and inferior solution set

First, put the maximum value of the positive index and the minimum value of the negative index of each level as the optimal solution set $V^{+}$. Similarly, put the minimum value of the positive index and the maximum value of the negative index of each level as the inferior solution set $V^{-}$.

$$
\begin{aligned}
V^{+} & =\left[\begin{array}{ccc}
v_{11}^{+} & \ldots & v_{1 m}^{+} \\
\ldots & \ldots & \ldots \\
v_{n 1}^{+} & \ldots & v_{m n}^{+}
\end{array}\right] \\
V^{-} & =\left[\begin{array}{ccc}
v_{11}^{-} & \ldots & v_{1 m}^{-} \\
\ldots & \ldots & \ldots \\
v_{n 1}^{-} & \ldots & v_{m n}^{-}
\end{array}\right]
\end{aligned}
$$

In Eq.(6) and Eq.(7), $v_{i j}^{+}$represents the optimal value of index $\mathrm{j}$ in level $\mathrm{i} . v_{i j}^{-}$represents the inferior value of index $\mathrm{j}$ in level $\mathrm{i}$.

(4) Determine the correlation degree between second-level index and the each level

First, calculate the proximity degree between the practical index value set and the optimal (inferior) set in each level.

$$
\begin{aligned}
& \left(D_{j}^{+}\right)_{-}=\sqrt{\sum_{i=1}^{m}\left(w_{i}\right)_{-}\left[\left(v_{i}-v_{i j}^{+}\right) /\left(v_{i j}^{+}-v_{i j}^{-}\right)\right]^{2}} \\
& \left(D_{j}^{+}\right)_{+}=\sqrt{\sum_{i=1}^{m}\left(w_{i}\right)_{+}\left[\left(v_{i}-v_{i j}^{+}\right) /\left(v_{i j}^{+}-v_{i j}^{-}\right)\right]^{2}} \\
& \left(D_{j}^{-}\right)_{-}=\sqrt{\sum_{i=1}^{m}\left(w_{i}\right)_{-}\left[\left(v_{i}-v_{i j}^{-}\right) /\left(v_{i j}^{+}-v_{i j}^{-}\right)\right]^{2}} \\
& \left(D_{j}^{-}\right)_{+}=\sqrt{\sum_{i=1}^{m}\left(w_{i}\right)_{+}\left[\left(v_{i}-v_{i j}^{-}\right) /\left(v_{i j}^{+}-v_{i j}^{-}\right)\right]^{2}}
\end{aligned}
$$

In Eq.(8) (11), $D_{j}^{+}=\left[\left(D_{j}^{+}\right)_{-},\left(D_{j}^{+}\right)_{+}\right]$represents the proximity degree interval between the practical index value set and the optimal set in level j. $D_{j}^{-}=\left[\left(D_{j}^{-}\right)_{-},\left(D_{j}^{-}\right)_{+}\right]$represents the proximity degree interval between the practical index value set and the inferior set in level $\mathrm{j}$.

Then, calculate the correlation degree between the practical index value set and the optimal (inferior) set in each level according to Eq.(12) and Eq.(13).

$$
\begin{gathered}
\rho\left(v, D_{j}^{+}\right)=\frac{\left(D_{j}^{+}\right)_{-}}{\left(D_{j}^{+}\right)_{-}+\left(D_{j}^{-}\right)_{-}}+\frac{\left(D_{j}^{+}\right)_{+}}{\left(D_{j}^{+}\right)_{+}+\left(D_{j}^{-}\right)_{+}} \\
\rho\left(v, D_{j}^{-}\right)=\frac{\left(D_{j}^{-}\right)_{-}}{\left(D_{j}^{+}\right)_{-}+\left(D_{j}^{-}\right)_{-}}+\frac{\left(D_{j}^{-}\right)_{+}}{\left(D_{j}^{+}\right)_{+}+\left(D_{j}^{-}\right)_{+}}
\end{gathered}
$$

In Eq.(12) and Eq.(13), $\rho\left(v, D_{j}^{+}\right)$represents the correlation degree between the practical index value set and the optimal set in level j. $\rho\left(v, D_{j}^{-}\right)$represents the correlation degree between the practical index value set and the inferior set in level $\mathrm{j}$. 
(5) Determine the membership degree between second-level index and each level

Calculate the membership degree according to Eq.(14).

$$
\begin{gathered}
K_{j}(U)=\frac{\rho\left(v, D_{j}^{+}\right)}{\rho\left(v, D_{j}^{+}\right)+\rho\left(v, D_{j}^{-}\right)}=\frac{\rho\left(v, D_{j}^{+}\right)}{2 \rho\left(v, D_{j}^{+}\right)-1} \\
K_{j_{0}}(U)=\max _{1 \leq j_{0} \leq n} K_{j}(U)
\end{gathered}
$$

According to the principle of maximum membership degree, the evaluation result is $j_{0}$.

(6) Multilevel extension rating

Using the index model and algorithm model above, we can evaluate the fist-level index through the combination between the evaluation result of second-level index and the weight of first-level index. Assume the evaluation result of second-level index $\mathrm{k}$ is $j_{k}\left(1 \leq j_{k} \leq n\right.$ ),we can calculate the evaluation result of the fisrt-level index using Eq.(16).

$$
j^{*}=\sum_{k=1}^{t} j_{k} \mu_{k}
$$

In Eq.(16), $j^{*}$ is the evaluation result of the fisrt-level index. $t$ represents the number of the first-level index. $\mu_{k}$ represents the weight of the first-level index.

\section{Case Analysis}

In this paper, we pick a typical area as an example to verify the feasibility of this algorithm. The evaluation result is divided into 5 grades, which are excellent, good, medium, qualified, and poor. According to the index system and the relevant data, we can get the actual data and the classical range of each index.

We ask ten expects to mark the relative importance of each index, and we can calculate the interval weight of each index according to the weight algorithm above. According to Eq.(6) (15), we can calculate the membership degree of the first-level degree. The results are as shown in

\begin{tabular}{|c|c|c|c|c|c|}
\hline $\begin{array}{c}\text { First-level } \\
\text { indov }\end{array}$ & Interval weight & Grade & $\begin{array}{c}\text { Maximum } \\
\text { momborchin domanom }\end{array}$ & $\begin{array}{c}\text { Second-level } \\
\text { indav }\end{array}$ & Interval weight \\
\hline \multirow{7}{*}{$\begin{array}{c}\text { Safety and } \\
\text { reliability }\end{array}$} & \multirow{7}{*}[0.5427,0.5904]{} & \multirow{7}{*}{$j=2$} & \multirow{7}{*}{2.4599} & $A_{1}$ & {$[0.1987,0.2268]$} \\
\hline & & & & $A_{2}$ & {$[0.1073,0.1429]$} \\
\hline & & & & $A_{3}$ & {$[0.0361,0.0436]$} \\
\hline & & & & $A_{4}$ & {$[0.1374,0.1663]$} \\
\hline & & & & $A_{5}$ & {$[0.0293,0.0323]$} \\
\hline & & & & $A_{6}$ & {$[0.3581,0.3761]$} \\
\hline & & & & $A_{7}$ & {$[0.0512,0.0618]$} \\
\hline \multirow{5}{*}{$\begin{array}{c}\text { Equipment } \\
\text { status }\end{array}$} & \multirow{5}{*}[0.2096,0.2608]{} & \multirow{5}{*}{$j=2$} & \multirow{5}{*}{1.7216} & $B_{1}$ & {$[0.0365,0.0375]$} \\
\hline & & & & $B_{2}$ & {$[0.0849,0.1013]$} \\
\hline & & & & $B_{3}$ & {$[0.0598,0.0725]$} \\
\hline & & & & $B_{4}$ & {$[0.4038,0.4527]$} \\
\hline & & & & $B_{5}$ & {$[0.3191,0.3605]$} \\
\hline \multirow{5}{*}{$\begin{array}{l}\text { Service } \\
\text { support }\end{array}$} & \multirow{5}{*}[0.1507,0.1804]{} & \multirow{5}{*}{$j=2$} & \multirow{5}{*}{1.4323} & $C_{1}$ & {$[0.0339,0.0349]$} \\
\hline & & & & $C_{2}$ & {$[0.0562,0.0801]$} \\
\hline & & & & $C_{3}$ & {$[0.2328,0.2652]$} \\
\hline & & & & $C_{4}$ & {$[0.1411,0.1700]$} \\
\hline & & & & $C_{5}$ & {$[0.4474,0.4708]$} \\
\hline
\end{tabular}
Tab.3.

Tab.3 Interval weight and evaluation results 
The results in Tab.3 show that the level of safety and reliability and the service support index is medium, and the level of equipment status is good. According to the weight of the first-level index and Eq.(16), the final evaluation result of power communication network in this typical area can be calculated, that is $j^{*}=[1.8060,2.0632]$. It can be seen that the final evaluation result is between excellent and medium, and it has a strong tendency to good.

From the analysis of the example, it can be seen that the new method for the diagnosis of power backbone communication network based on extension rating can evaluate the power communication network from the whole angle. Moreover, the method can also evaluate the safety and reliability, the equipment status and the service support, so as to provide theory principle to the construction of the power communication network.

\section{Conclusion}

This paper studied the power communication network and built an index system of the power communication network. In addition, this paper used the interval weight to reduce the subjectivity of the weight and proposed a new method for the diagnosis of power backbone communication network based on extension rating. It can be seen that this method can evaluate the power communication network and has a great significance to the construction of the power communication network.

Financial aid supplement: National Natural Science Foundation of China No.51507063

\section{References}

[1]Erzhao Bian. Regional power network development level diagnosis system and its application [D]. North China Electric Power University, 2014

[2]Runze Wu, Xinyu Wang, Xiaoping Zhang, Wenxia Liu. Adaptive evaluation method for distributed power network monitoring and control system [J]. power system automation, 2014,08:72-77.

[3]Alios Ye, EnGuo Zhu, Qian Cheng, Hongwei Zhang. Electrical equipment safety assessment for improved interval analytic hierarchy analysis method [J]. Automation of electric power systems of, 2015,01:32-36.

[4]Bin Li, Yalong Wang. Evaluation of power grid technology, [J].2015,04:1146-1152.

[5]Yan Bin Li, Xinyi Yu, Zhijie Wang. The grey correlation analysis and TOPSIS method of photovoltaic power generation project risk assessment [J]. Power system technology, 2013,06:1514-1519. 